\title{
Thermal Management of Lithium-Ion Battery using Heat Pipes
}

\author{
Jong Wook Yoon', Dong Soo Jang1, Sungho Yun', Hyun Ho Shin', Yongchan Kim² \\ ${ }^{1,1,1,1}$ Graduate School of Mechanical Engineering, Korea University \\ Seoul 136-713, Republic of Korea \\ nim_yoon@korea.ac.kr; nicebb0y@korea.ac.kr, hadogo@korea.ac.kr, neurochall@korea.ac.kr \\ ${ }^{2}$ Department of Mechanical Engineering, Korea University \\ Seoul 136-713, Republic of Korea \\ yongckim@korea.ac.kr
}

\section{Extended Abstract}

This study presents the heat transfer characteristics of U-shaped heat pipes with asymmetric channels, which improve a non-uniform temperature distribution in a battery. An lithium-ion battery system generally adopts a forced convection cooling method using a fan owing to low power consumption and cost. The forced convection cooling can reduce a temperature increase in the battery. However, the non-uniform distribution of the temperature on the surface of the battery is inevitable, which affects battery life and performance $[1,2]$. The proposed heat pipes are made of aluminum plate with grooved wick. The U-shape consists of three types of channel designs with different asymmetric ratios. The asymmetric ratios of the channel lengths are $0.4: 1,0.5: 1$, and 0.6: 1 . Through CFD simulations, the non-uniform temperature distribution of the battery in the 'fan alone' condition and the 'fan + duct' condition are investigated, and the experiment under the condition of the 'fan + heat pipe' is carried out. The results shows that the ratio of the asymmetric channel and the heat pipe position in the battery critically affect the non-uniform temperature distribution. In addition, it is concluded that the fan air flow rate can be controlled by the asymmetric channel structure of the heat pipe.

\section{References}

[1] M. Wu, K. H. Liu, Y. Wang, C. Wan, "Heat dissipation design for lithium-ion batteries," Journal of Power Sources, vol. 109, pp. 160-166, 2002.

[2] S. C. Chen, C. C. Wan, Y. Y. Wang, "Thermal analysis of lithium-ion batteries," Journal of Power Sources, vol. 140, pp. 111-124, 2005. 